ज्ञ FRANÇAISE

$>\mathrm{DE}$

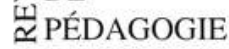

\section{Revue française de pédagogie}

Recherches en éducation

171 | avril-juin 2010

La mixité scolaire : une thématique (encore)

d'actualité?

\title{
Valeurs et attentes des collégiens et des collégiennes envers l'école : une mixité à construire
}

Middle-school students' expectations of their schooling: Setting-up coeducation Valores y expectativas de los alumnos y de las alumnas de colegio para con la escuela: una mixidad por construir

Werte und Erwartungen der College-SchülerInnen : eine noch zu bildende Koedukation

\section{Claire Safont-Mottay, Nathalie Oubrayrie-Roussel et Yves Prêteur}

\section{(2) OpenEdition}

Journals

Édition électronique

URL : http://journals.openedition.org/rfp/1889

DOI : $10.4000 / \mathrm{rfp} .1889$

ISSN : 2105-2913

Éditeur

ENS Éditions

Édition imprimée

Date de publication : 1 juillet 2010

Pagination : 31-45

ISBN : 978-2-7342-1187-7

ISSN : 0556-7807

Référence électronique

Claire Safont-Mottay, Nathalie Oubrayrie-Roussel et Yves Prêteur, " Valeurs et attentes des collégiens et des collégiennes envers l'école : une mixité à construire », Revue française de pédagogie [En ligne], 171 | avril-juin 2010, mis en ligne le 01 juin 2014, consulté le 19 avril 2019. URL : http:// journals.openedition.org/rfp/1889; DOI : 10.4000/rfp.1889 


\title{
Valeurs et attentes des collégiens et des collégiennes envers l'école : une mixité à construire
}

\author{
Claire Safont-Mottay, \\ Nathalie Oubrayrie-Roussel \\ et Yves Prêteur
}

L'objectif de cette étude est d'appréhender les valeurs et attentes à l'égard de l'école et les modes différenciés d'investissement chez les garçons et les filles en fin de collège. L'étude concerne 681 collégiens scolarisés en classe de $3^{e}$ (générale et SEGPA), interrogés par questionnaire à propos de leur rapport à l'école et aux savoirs scolaires, des valeurs qu'ils accordent à l'école et du sens qu'ils attribuent à leur scolarité, en relation avec leurs perceptions des attentes et actions éducatives. Alors que les filles et les garçons présentent des similitudes du point de vue des valeurs qu'ils accordent à l'école et du sens accordé à l'expérience scolaire, l'étude montre qu'ils développent des stratégies d'investissement différentes. Les filles (en filière générale), plus investies dans les relations sociales et communicatives, mettent en avant des valeurs de socialisation et des buts de sociabilité favorisant chez elles l'engagement envers les apprentissages et une meilleure performance. Les différences observées entre garçons et filles quant aux modes de sociabilité et à la réussite scolaire sont discutées à la lumière de travaux portant sur la socialisation différentielle.

Descripteurs (TESE) : adolescent, environnement socioculturel, sexe, parcours scolaire, éducation mixte.

\section{INTRODUCTION}

Les débats sur les multiples enjeux de la mixité scolaire en rapport avec l'égalité des chances à l'école (Marry, 2004 ; Duru-Bellat \& Marin, 2009) d'une part, l'évolution du rapport à l'école et aux savoirs scolaires des jeunes en raison de la situation concurrentielle avec les médias et les nouvelles technologies (Pasquier, 2005) d'autre part nous incitent à nous interroger sur la signification de l'école et sur le coût psychologique de l'investissement scolaire pour chaque adolescent. Le collège est-il reconnu par l'élève pour sa fonction de transmission de savoirs actualisables dans le présent et/ou nécessaires pour sa vie future (valeur scolaire ou instrumentale)? Est-il un lieu essentiel pour le développement des relations sociales actuelles (valeur sociale) ? Les attentes à l'égard de l'institution scolaire diffèrent-elles pour les filles et les garçons? La première partie de cet article est consacrée à la présentation des éléments du contexte dans lequel s'inscrit la question de la différenciation des trajectoires scolaires des filles et des garçons. 
La seconde partie présente la procédure et les résultats d'une étude empirique réalisée auprès de 681 collégiens de $3^{\mathrm{e}}$.

\section{DIFFÉRENCES DE SEXE À L'ÉCOLE ET SOCIALISATION DIFFÉRENCIÉE}

Bien avant la naissance, les garçons et les filles font l'objet d'appréciations et d'attentes différenciées de la part de leur entourage familial. La famille, principal agent de socialisation pendant l'enfance, a un rôle central dans la construction de l'identité sexuée. Les parents projettent sur leur enfant les traits qu'ils considèrent comme féminins ou masculins (couleurs, habillement, jouets, etc., voir Rouyer, 2007). Des pratiques de socialisation différentes selon le sexe se manifestent dans divers domaines tels que les interactions, le langage, les conduites motrices, l'expression des émotions, le jeu ou les apprentissages cognitifs (Rouyer \& Zaouche-Gaudron, 2006). L'enfant puis l'adolescent intègre les informations plurielles provenant de ses différents milieux de vie. Mais, en tant qu'acteur de sa construction identitaire sexuée, il ne se contente pas d'intérioriser des pratiques sexuées, des stéréotypes, des rôles, des statuts ou des places sexués et de se les approprier sur le plan cognitif. II s'en sert et les réoriente en fonction de son histoire personnelle et des contextes successifs dans lesquels il se développe et interagit avec des « autrui » différenciés et différenciateurs, mais aussi en fonction des déséquilibres entre les places sexuées qu'il occupe et des contradictions internes à ses partenaires éducatifs (Tap \& Zaouche-Gaudron, 1999). En effet, la famille n'est pas le seul milieu de vie, dans lequel l'enfant est inséré, qui soit marqué par la différenciation de sexe. Le milieu scolaire, de par son organisation, les interactions entre élèves et les interactions entre élèves et enseignants, constitue aussi un lieu de socialisation implicitement différenciateur selon le sexe (Zaidman, 1996 ; Duru-Bellat, 2005 ; Dafflon Novelle, 2006). Prenant le relais de la socialisation différenciée engagée dans le milieu familial, l'école « fait vivre aux filles et aux garçons des expériences différentes où se construisent et se transforment des identités sexuées qui contribuent à "fabriquer" conjointement avec de nombreuses autres influences, des différences de cursus scolaires, universitaires et professionnels » (Mosconi, 1999, p. 86). Rappelons que la variable sexe constitue le deuxième facteur de différenciation scolaire, après l'origine sociale: les filles s'investissent mieux à l'école; les garçons quant à eux réinvestissent mieux leur bagage scolaire dans leur carrière professionnelle ; les écarts entre filles et garçons décroissent à mesure que l'origine sociale s'élève (Baudelot \& Establet, 2007).

Selon leur genre, les élèves ne rencontrent pas les mêmes difficultés aux mêmes moments du cursus scolaire. Potvin, Paradis et Pouliot (2000), examinant les attitudes des enseignants de maternelle envers les enfants, montrent que les filles sont souvent perçues plus positivement que les garçons et considérées comme plus intelligentes, tranquilles, motivées, aidantes, responsables et conformistes. Au début de la scolarité, le cadre scolaire et ses exigences semblent plus favorables aux filles. Elles rencontrent moins de difficultés d'adaptation que les garçons. Les comportements féminins prescrits étant en bonne adéquation avec les attentes de l'institution (obéissance, soumission, calme, conformisme, attention, contrôle de soi...), elles exercent correctement leur métier d'élève. Ces comportements facilitant la tâche des enseignants, les filles font alors de leur part l'objet d'un biais évaluatif favorable qui est cependant limité aux aspects à la fois comportementaux et disciplinaires (Duru-Bellat, 2005 ; Ferrez, 2006). Ainsi le fait que les filles ne posent pas problème - en tout cas dans le système de représentation des maîtres - les dessert en même temps. En effet, en partie à cause de cette facilité qu'elles ont à endosser le rôle d'élève, elles deviennent moins visibles dans la classe et captent moins l'attention des enseignants qui, de fait, interagissent moins avec elles. L'école de la différence vaut pour les deux sexes. Les garçons affrontent quant à eux à une contradiction entre les caractéristiques masculines socialement reconnues (indépendance, agressivité, comportements moteurs vifs) et les normes et obligations scolaires (Felouzis, 1993). En fait ce conflit, entre les modèles de genre et le modèle scolaire, se résout généralement par une attention particulière de la part des enseignants à l'égard des garçons, perçus comme des élèves à fort potentiel qui se révéleront ultérieurement dans la scolarité. Sans en être conscients, les enseignants mobilisent des schèmes stéréotypés sur les qualités scolaires respectives des filles et des garçons.

Les recherches montrent également que les stéréotypes de sexe véhiculés par les enseignants ont des répercussions sur l'estime de soi des élèves (Mosconi, 2001 ; Marry, 2003 ; Jarlégan \& Tazouti, 2007) et, de fait, sur leur expérience scolaire (Dafflon Novelle, 2006). Ainsi ces stéréotypes amènent les filles à douter plus fortement de leurs capacités intellectuelles, ce qui renforce chez elles un moindre sentiment de 
valeur personnelle. Si ce questionnement et cette interrogation sont souvent perçus comme une vulnérabilité, comme l'analyse Raïd (2009), cette attitude renvoie à la problématique du "care » (souci des autres, sensibilité et responsabilité). Tronto (2006, cité par Raïd, 2009) suggère de réviser notre représentation de l'autonomie et, plus largement, des relations de l'être humain à ses vulnérabilités, en assumant ce que le care met au grand jour: "II ne s'agit pas d'une reconnaissance demandée à autrui, mais d'une reconnaissance de soi à soi » (Raïd, 2009, p. 83), d'une posture réflexive dont certains des enjeux peuvent être favorables à la réussite scolaire et dont le contraire est le déni. Cette voie nous permet de comprendre que nous sommes tous vulnérables (à ne pas confondre avec fragiles!).

La prédominance paradoxale des filles dans la réussite et l'adaptation scolaire a été maintes fois soulignée (Zazzo, 1993 ; Duru-Bellat, 2005 ; Baudelot \& Establet, 2007 ; MEN, 2008). Selon Terrail (1992a, 1997), la « surscolarisation » des filles est inhérente à leur investissement scolaire différentiel et s'explique en partie par une structuration historique des rapports de sexe, qui a longtemps tenu les femmes éloignées du pouvoir et de la concurrence marchande, les incitant à construire une vision moins compétitive de l'activité scolaire. Quant à leurs rapports plus faciles avec les enseignants, ils s'étayent sur la relation (elle-même plus facile) qu'elles ont avec leurs parents, fondée sur la confiance et la circulation de la parole. Enfin on peut relever plus souvent chez elles un effet propre de mobilisation personnelle qui serait au principe de leur intérêt pour l'appropriation des savoirs, de leurs attentes différenciées à l'égard des enseignants, de leur application au travail et de leur plaisir à l'école, assez fortement ancrés pour résister à l'expérience du redoublement.

La construction différenciée de l'identité psychosexuelle et sociale des filles et des garçons, fortement orientée par les stéréotypes sociaux, contribuerait pour un certain nombre d'auteurs à expliquer une plus grande réussite scolaire chez les filles et une plus grande démobilisation chez les garçons (CourtinatCamps \& Prêteur, 2010). Les stéréotypes, transmis aux adolescent-e-s et intégrés par eux au cours de la socialisation, susciteraient des valeurs et encourageraient des attitudes et des comportements nettement différenciés selon le genre. Certains de ces stéréotypes de genre entreraient en contradiction avec les attentes et exigences de l'institution scolaire. Le fait de rompre avec les "stéréotypes sexués " ou de s'affranchir des rôles sexués pourrait avoir des effets sur la performance scolaire des élèves (Chevet, 2006). L'affranchissement des stéréotypes de sexe s'accompagnerait d'une meilleure réussite scolaire, cet affranchissement serait plus marqué dans les milieux favorisés que dans les milieux modestes et chez les filles que les garçons (dans une proportion du simple au double, voir notamment Bouchard, Saint-Amant \& Tondreau, 1998).

L'intérêt actuel de la recherche tend également à se porter sur la socialisation par les pairs en milieu scolaire, notamment à l'adolescence (Dubet \& Martuccelli, 1996 ; van Zanten, 2001 ; Claes, 2003 ; Coslin, 2007), pour tenter de comprendre et d'expliquer la différenciation des conduites scolaires et le rapport à l'école des adolescents et des adolescentes. Les différences constatées dans les relations entre enseignants et élèves, parents et enfants s'observent également dans les relations des élèves entre eux. Les enfants reprennent à leur propre compte les comportements et les attitudes appris dans la famille, dans les médias, dans leurs jeux, à l'école, et ceci d'autant plus que les adultes ne les découragent pas (Forest, 1992). Dans le temps des remaniements de l'adolescence, l'insertion du jeune dans le collège offre un environnement social plus étendu et de multiples possibilités d'interaction sociale avec des groupes de pairs différenciés (Mallet, 2003). Malgré une majorité de travaux qui recensent un effet protecteur des amitiés devant le mal-être scolaire (Berndt, Laychak \& Park, 1990 ; Ladd, 1990), d'autres témoignent de la nécessité de prendre en considération les caractéristiques du groupe de pairs (Fuligni et al., 2001 ; Michinov, 2004). Les enfants et adolescents sélectionnent leurs amis en fonction de leurs attitudes, de leurs valeurs, de leurs centres d'intérêt, mais également en fonction de leurs compétences et de leurs aspirations scolaires. L'appartenance à un groupe est fondamentale et répond à deux types de besoins: le besoin d'être reconnu par les autres, d'exister aux yeux des autres et le besoin de sécurité, de se sentir protégé dans et par le groupe. En effet, au contact des pairs, s'élabore une image de groupe, des signes d'appartenance qui nient la singularité de l'individu pour valoriser le «nous", source de sécurité et de protection devant l'angoisse existentielle (Coslin, 2007). Ainsi, plus les difficultés scolaires augmentent et réduisent le sentiment de proximité à l'égard de l'école, plus la relation au groupe de pairs représente un contrefort symbolique rassurant et recherché (Millet \& Thin, 2005). Se sentant exclus, les élèves en difficulté scolaire recherchent souvent une reconnaissance par les pairs qui sont les plus éloignés des normes sociales et des règles scolaires (Fuligni et al., 2001). 


\section{DIFFÉRENCIATION DU RAPPORT À L'ÉCOLE CHEZ DES COLLÉGIENS DE TROISIĖME}

L'enquête dont nous allons rendre compte s'est donnée pour objectif de répondre à la question suivante: en fin de premier cycle secondaire, filles et garçons élaborent-ils des attentes et des modes d'investissement différenciés selon leur expérience scolaire, leur parcours et leur orientation scolaires (filière générale ou filière professionnelle adaptée) ? Plusieurs registres peuvent être convoqués pour caractériser le rapport de l'adolescent à l'apprendre. Ils contribuent à signifier le mode d'investissement à l'école. En se fondant sur un recueil d'informations par questionnaire auprès de collégiens de $3^{\mathrm{e}}$, il s'agit d'analyser comment ces adolescents construisent de façon différenciée :

- tout d'abord, leur rapport à l'école. En effet, la formation et l'expérience scolaire ne sauraient être pensées seulement en termes de transmission et de réception de savoirs, de connaissances et de compétences, mais aussi en termes de développement (Rochex, 1995). Cependant cette problématique développementale prend en compte fréquemment le développement intellectuel et cognitif, et plus rarement le développement et la transformation de soi sur le registre affectif et subjectif. Nous rejoignons la posture explicative de Malrieu (1980) et de Tap (1985) : la construction de l'identité est traversée par des doutes, des épreuves, des conflits, des crises, des remaniements, parfois même des renoncements, et des dépassements, constitutifs des choix et des conduites de chaque personne (adolescente dans ce cas), pour se déterminer en fonction des attentes et contraintes de ses milieux de vie et de l'école en particulier ;

- en second lieu, il s'agit d'analyser comment ils construisent différents types de représentations de l'école focalisées sur des valeurs accordées à celle-ci. La culture scolaire constitue un réseau de significations et de connaissances dans lequel règles, valeurs, croyances, représentations, savoirs et savoir-faire sont cristallisés (Prêteur, Constans \& Féchant, 2004). La notion de valeur renvoie au fait, pour un sujet, de reconnaître le rôle de l'école comme important dans la réalisation de ses objectifs (Léonardis \& Prêteur, 2007). La valeur que l'élève accorde à l'école nous paraît particulièrement importante pour mieux comprendre la manière dont l'élève, porteur d'une histoire, se pense comme apprenant et conçoit les savoirs et leurs finalités. Par la notion de valeur accordée à l'école, il faut principalement comprendre la relation de sens, donc de valeur, entre un individu (ou un groupe) et l'école comme lieu, ensemble de situations et de personnes (Charlot, Bautier \& Rochex, 1992).

Nous faisons l'hypothèse que la valeur accordée à l'école et la mobilisation à l'école jouent un rôle important dans l'engagement envers les apprentissages et, par conséquent, sur la réussite scolaire chez les collégien-ne-s de $3^{e}$.

\section{Population}

Nous avons constitué un échantillon de 681 collégiens de $3^{\mathrm{e}}$ doublement dichotomisé, d'une part en confrontant garçons et filles, et d'autre part en différenciant la filière générale $(n=563)$ et les sections d'enseignement général et professionnel adapté (1) (SEGPA, $n=118$ ). Cette deuxième dichotomie paraît justifiée dans la mesure où la littérature et nos propres travaux antérieurs laissent supposer des parcours et des projets typés (Dumora, 2001 ; Jellab, 2001 ; Léonardis, Capdevielle \& Prêteur, 2006 ; DuruBellat \& van Zanten, 2009).

Parmi les élèves inscrits en $3^{\mathrm{e}}$ générale, on dénombre 322 garçons $(57,2 \%)$ et 241 filles $(42,8 \%)$ âgés de 13 à 17 ans (voir en annexe 1). Les élèves en $3^{\text {e }}$ SEGPA, 69 garçons $(58,5 \%)$ et 49 filles $(41,5 \%)$, sont âgés de 14 à 17 ans. Les adolescents sont tous scolarisés dans différents collèges urbains et suburbains de Midi-Pyrénées et issus de milieux socioculturels diversifiés. La catégorie des parents occupant des emplois peu qualifiés est plutôt surreprésentée dans notre échantillon $(57,5 \%$ des mères et $60,5 \%$ des pères dans l'échantillon total ; $52,7 \%$ des mères et $57,1 \%$ des pères pour les élèves de filière générale ; $83,5 \%$ des mères et $79,5 \%$ des pères pour les élèves en SEGPA). $38 \%$ des mères et $44 \%$ des pères ont un faible niveau d'études (études primaires, $3^{e}$, CAP ou BEP) dans l'échantillon total ; $33,6 \%$ des mères et $41,4 \%$ des pères pour les élèves de filière générale; $68,9 \%$ des mères et $64,3 \%$ des pères pour les élèves en SEGPA.

\section{Instrument de recueil des données}

Le questionnaire auquel les adolescents ont répondu au cours du troisième trimestre de l'année scolaire est issu d'un protocole plus large sur "les jeunes, l'école et leur avenir" (Prêteur, Constans \& Féchant, 2004). Notre instrument se compose de trois rubriques. La première concerne la trajectoire scolaire : 
- 15 questions se rapportent au « parcours scolaire de l'élève " (classe dans laquelle il a le plus ou le moins travaillé, redoublement ou absence de redoublement, classe redoublée, saut de classe, moyenne générale des trois trimestres scolaires de l'année de $3^{e}$, satisfactions personnelle et parentale concernant les résultats de l'année scolaire) ;

- 8 questions se rapportent au « vécu au collège »; elles sont relatives à l'absentéisme, aux retards, aux difficultés rencontrées au collège, à l'aide scolaire et à l'acceptation de l'école et de son organisation.

La deuxième rubrique porte sur le rapport à l'école : dans cette rubrique, relative à l'expérience subjective du sujet en tant qu'élève, les valeurs accordées à l'école et le sentiment de proximité scolaire sont étudiés. Les valeurs accordées à l'école ont été appréhendées à partir d'une échelle composée de 20 items portant sur la représentation de l'école (« Ce que je veux que l'école m'apporte ", voir Léonardis \& Prêteur, 2007 ; voir également l'annexe 2). Les réponses se présentent sous la forme d'une échelle de type Lickert en 4 points, allant de «faux» (1 point) à «vrai » (4 points), en passant par "plutôt faux » (2 points) et "plutôt vrai » (3 points). L'instrument permet de spécifier des valeurs relevant d'une représentation plus instrumentale de l'école comme lieu de formation, par contraste avec des valeurs de socialisation attendues par l'élève (Léonardis \& Prêteur, 2007). Les analyses psychométriques (calcul du coefficient de cohérence interne, l' $\alpha$ de Cronbach, réalisation d'une analyse factorielle en composantes principales avec rotation Varimax) ont conduit à un travail de sélection et de répartition des items. Suite à ces analyses, nous retenons une sélection de 13 items renvoyant à trois dimensions (regroupement des items sur trois facteurs de l'ACP représentant $50 \%$ de la variance totale, $20 \%$ pour le premier, $17 \%$ pour le second et $13 \%$ pour le troisième, $\mathrm{KMO}=0,74)$. La première dimension $(\alpha=0,73)$ regroupe les items relatifs à des valeurs scolaires relevant d'une représentation instrumentale de l'école comme lieu de formation. La deuxième dimension correspond aux valeurs sociales attendues par l'élève $(\alpha=0,64)$ renvoyant typiquement aux relations avec les pairs et à la recherche d'affinités. La dernière dimension $(\alpha=0,63)$ regroupe des buts relevant d'une recherche de conformité aux attentes des adultes, vis-à-vis de la réussite en classe, et à la désirabilité sociale. L'analyse de la structure de l'échelle met ainsi en évidence une répartition fine des items distinguant des valeurs scolaires, des valeurs sociales et des valeurs hétéronomes.
II conviendra néanmoins d'interpréter avec précaution les résultats au regard des indices de cohérence interne satisfaisants, sans pour autant être très élevés ( $\alpha$ de Cronbach allant de 0,63 à 0,73).

Dix items sont relatifs au sentiment de proximité/ distance vis-à-vis de la scolarité (apprentissages, relation avec l'institution scolaire, attentes des enseignants et des parents, pour lesquels $\alpha=0,65)$. Ils permettent d'apprécier notamment le mal-être de l'élève et ses difficultés à trouver ou donner du sens à ses activités ("Quand je suis au collège, je me demande parfois ce que je fais ", "Je ne comprends pas ce que les enseignants attendent de moi », «Au niveau du travail scolaire, je fais juste ce qu'on me demande, sans aller au-delà, sans approfondir », etc.). Le questionnement du sens de la scolarité par les adolescents et de leurs perceptions des attentes et actions éducatives renvoie à la notion de (dé)mobilisation. Celle-ci se conçoit comme une activité essentiellement interne par laquelle le sujet donne sens à son expérience scolaire. Dans une dernière rubrique sont rassemblées des informations sociobiographiques sur la famille de l'adolescent interrogé.

\section{UN INVESTISSEMENT SCOLAIRE DIFFÉRENCIÉ DES FILLES ET DES GARÇONS}

Le genre représente un facteur de différenciation en milieu scolaire. Nous allons donc analyser nos résultats au regard de cette variable de contrôle, ceci afin d'appréhender la manière dont les adolescents, filles et garçons, se positionnent à l'égard de l'école.

\section{Des parcours scolaires différenciés entre collégiens}

Nous avons dans un premier temps comparé le parcours scolaire et le vécu des collégiens en distinguant le genre et la filière suivie.

\section{Filles et garçons ont-ils suivi le même parcours} scolaire?

Dans le tableau 1 sont présentés les taux de redoublement et de saut de classe, ainsi que la proportion de moyennes des notes (moyennes générales des notes aux trois trimestres) supérieures à 10 .

Les tests de khi 2 réalisés sur les indicateurs du parcours scolaire ont permis de mettre au jour des différences de genre significatives au sein de la population 
Tableau 1. - Éléments du parcours scolaire

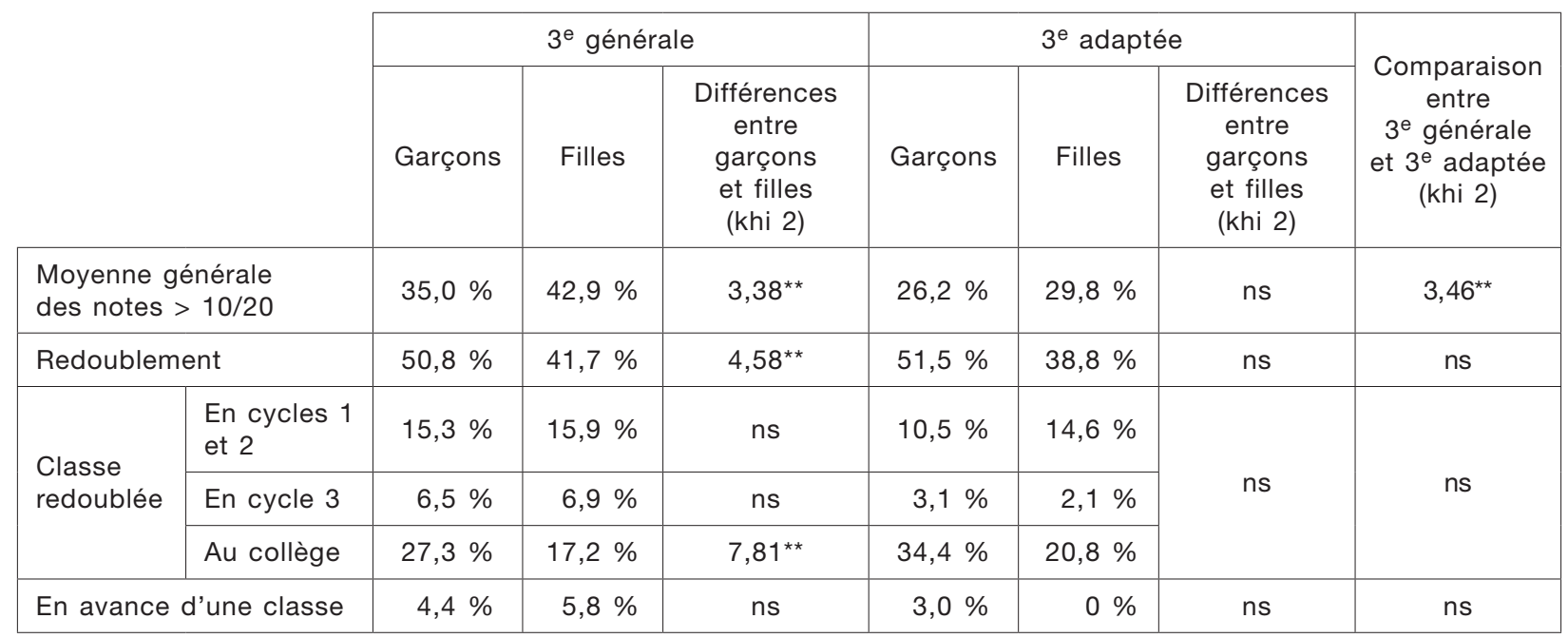

Note : ** significatif à $\mathrm{p}<0,05$; ns pour non significatif.

de $3^{\mathrm{e}}$ générale. Ainsi les filles ont de meilleures notes (khi $2=3,38 ; p<0,05$ ). Elles ont également redoublé moins souvent que les garçons (khi $2=4,58$; $p<0,05$ ), alors que les garçons ont redoublé plus fréquemment au collège que les filles (khi $2=7,81$; $p<0,05)$. Aucune différence entre les sexes n'est mise en évidence pour les élèves de SEGPA.

L'évaluation de la moyenne générale est supérieure chez les élèves de $3^{\mathrm{e}}$ générale (khi $2=3,46 ; p<0,05$ ), sachant toutefois que les moyennes ne sont pas directement comparables d'une filière à l'autre. Cependant les différences de trajectoire scolaire entre les filières restent faibles. Les élèves de $3^{e}$ générale ont été tout autant confrontés au redoublement que ceux de SEGPA, en primaire comme au collège. Si le taux de redoublement distingue peu les élèves de section adaptée de leurs camarades de filière générale, c'est en revanche le milieu social d'origine qui oppose les adolescents. Les catégories socioprofessionnelles les plus défavorisées (2) (les ouvriers, qualifiés, non qualifiés, agricoles, les retraités employés ou ouvriers et les personnes sans activité professionnelle) sont surreprésentées en filière SEGPA (profession de la mère : khi $2=41,9$; ddl $=3 ; p<0,000$; profession du père : khi $2=20,9 ; d d l=3 ; p<0,000$ ). Les scolarités les plus faibles (non scolarisé et niveau primaire, scolarisé de la $6^{\mathrm{e}}$ à la $3^{\mathrm{e}}$ ) sont également surreprésentées (scolarité de la mère: khi $2=34,6$; $\mathrm{ddl}=3 ; \mathrm{p}<0,000 ;$ scolarité du père : khi $2=19,4$; $d d l=3 ; p<0,000)$.
Une analyse de régression logistique binaire (3) a été réalisée dans le but d'identifier les facteurs qui caractérisent le mieux les élèves de $3^{e}$ SEGPA par rapport aux élèves de $3^{\mathrm{e}}$ générale. L'origine socioprofessionnelle, le niveau d'études des parents, ainsi que les éléments du parcours scolaire pour lesquels l'étude exploratoire a mis en évidence des différences entre les filières (comme la moyenne des notes et le taux de redoublement, cf. tableau 1) ont été entrés comme variables explicatives. L'évaluation statistique de la régression montre que, parmi toutes les variables explicatives prises en compte, c'est la scolarité de la mère qui différencie le mieux les deux filières (Wald $=19,09 ; p<0,00 ; \exp (B)=9,52 ; 90 \%$ des observations sont bien classées).

Filles et garçons ont-ils le même vécu au collège ?

Dans le tableau 2, nous comparons les réponses des filles et des garçons de $3^{\mathrm{e}}$ générale et de $3^{\mathrm{e}}$ adaptée à trois questions relatives au bien-être et au mal-être scolaire: le plaisir à aller au collège et l'absentéisme.

Notons auprès de notre échantillon de collégiens de $3^{\mathrm{e}}$ générale les répartitions voisines des observations effectuées par Terrail (1992b) : 54,6\% des garçons pour $66,7 \%$ des filles déclarent aimer aller au collège. Ainsi les filles « aiment plus aller au collège ", en particulier celles qui sont scolarisées en $3^{e}$ générale (khi $2=7,82 ; p<0,01$ ). Ces réponses restent néanmoins soumises au biais de désirabilité sociale, 
Tableau 2. - Vécu au collège

\begin{tabular}{|c|c|c|c|c|c|c|c|}
\hline & \multicolumn{3}{|c|}{$3^{\mathrm{e}}$ générale } & \multicolumn{3}{|c|}{$3^{e}$ adaptée } & \multirow{2}{*}{$\begin{array}{l}\text { Comparaison } \\
\text { entre } \\
3^{e} \text { générale } \\
\text { et } 3^{e} \text { adaptée } \\
\text { (khi 2) }\end{array}$} \\
\hline & Garçons & Filles & $\begin{array}{l}\text { Différences } \\
\text { entre garçons } \\
\text { et filles } \\
\text { (khi 2) }\end{array}$ & Garçons & Filles & $\begin{array}{l}\text { Différences } \\
\text { entre garçons } \\
\text { et filles } \\
\text { (khi 2) }\end{array}$ & \\
\hline $\begin{array}{l}\text { En général, aimes- } \\
\text { tu aller au collège ? }\end{array}$ & $54,6 \%$ & $66,7 \%$ & $7,82^{\star * *}$ & $60,0 \%$ & $76,2 \%$ & $2,99^{*}$ & ns \\
\hline $\begin{array}{l}\text { T'arrive-t-il } \\
\text { de sécher } \\
\text { des cours? }\end{array}$ & $16,8 \%$ & $15,4 \%$ & ns & $18,6 \%$ & $22,4 \%$ & ns & ns \\
\hline $\begin{array}{l}\text { T'arrive-t-il d'être } \\
\text { assez souvent } \\
\text { absent? }\end{array}$ & $20,6 \%$ & $16,7 \%$ & ns & $20,3 \%$ & $18,4 \%$ & ns & ns \\
\hline
\end{tabular}

Note : * significatif à $\mathrm{p}<0,10 ;{ }^{* \star *}$ significatif à $\mathrm{p}<0,01$.

Tableau 3. - Moyennes et écarts types de la valeur accordée à l'école (test t)

\begin{tabular}{|c|c|c|c|c|c|c|c|}
\hline & \multicolumn{3}{|c|}{$3^{e}$ générale } & \multicolumn{3}{|c|}{$3^{e}$ adaptée } & \multirow{2}{*}{$\begin{array}{c}\text { Comparaison } \\
\text { entre } \\
3^{e} \text { générale } \\
\text { et } 3^{\mathrm{e}} \text { adaptée } \\
\text { (test } \mathrm{t} \text { ) }\end{array}$} \\
\hline & Garçons & Filles & $\begin{array}{l}\text { Différences } \\
\text { entre garçons } \\
\text { et filles (t) }\end{array}$ & Garçons & Filles & $\begin{array}{l}\text { Différences } \\
\text { entre garçons } \\
\text { et filles (t) }\end{array}$ & \\
\hline Valeur scolaire & $\begin{array}{c}20,3 \\
(\sigma=3,3)\end{array}$ & $\begin{array}{c}20,4 \\
(\sigma=3,1)\end{array}$ & ns & $\begin{array}{c}20,5 \\
(\sigma=3,2)\end{array}$ & $\begin{array}{c}19,9 \\
(\sigma=3,1)\end{array}$ & ns & ns \\
\hline Valeur sociale & $\begin{array}{c}17,2 \\
(\sigma=2,7)\end{array}$ & $\begin{array}{c}17,0 \\
(\sigma=2,6)\end{array}$ & ns & $\begin{array}{c}17,0 \\
(\sigma=2,7)\end{array}$ & $\begin{array}{c}16,9 \\
(\sigma=2,6)\end{array}$ & ns & ns \\
\hline Valeur de conformité & $\begin{array}{c}9,3 \\
(\sigma=2,4)\end{array}$ & $\begin{array}{c}9,1 \\
(\sigma=2,2)\end{array}$ & ns & $\begin{array}{c}9,4 \\
(\sigma=2,4)\end{array}$ & $\begin{array}{c}9,4 \\
(\sigma=2,2)\end{array}$ & ns & ns \\
\hline
\end{tabular}

sachant que les jeunes ont été interrogés en milieu scolaire. Contrairement à nos travaux précédents auprès de collégiens (Oubrayrie-Roussel \& SafontMottay, 2007), aucun lien significatif n'est observé entre le sexe et le fait de sécher les cours $(16,7 \%$ des répondants, garçons et filles confondus). II en est de même pour le fait d'être souvent en retard $(20,5 \%$ des répondants, garçons et filles confondus).

\section{Rapport à l'école et réussite scolaire}

Nous allons tester l'hypothèse selon laquelle le rapport à l'école joue un rôle important dans l'engagement envers les apprentissages et, par conséquent, sur la réussite scolaire chez les collégien-ne-s. Nous supposons également que ce rôle sera relatif à leur sexe. Les résultats de l'étude exploratoire et descriptive des deux dimensions du rapport à l'école: la valeur accordée à l'école et le sentiment de proximité/ distance sont tout d'abord présentés. Puis des analyses de régressions multiples permettent de tester le modèle explicatif de la réussite scolaire et de vérifier l'existence de différences intersexes.

\section{Valeur accordée à l'école}

L'élève se rend-il à l'école parce qu'il a conscience de l'importance de cette dernière pour son avenir ou bien parce qu'il y est obligé et se plie aux exigences sociétales (Courtinat-Camps \& Prêteur, 2010) ? Afin de répondre à cette question, nous avons interrogé les élèves sur les valeurs qu'ils accordaient à l'école. Le tableau 3 regroupe les observations de l'étude ainsi que les résultats des tests t permettant de comparer 
Tableau 4. - Moyennes et écarts types du sentiment de proximité/distance vis-à-vis de la scolarité

\begin{tabular}{|c|c|c|c|c|c|c|c|}
\hline & \multicolumn{3}{|c|}{$3^{e}$ générale } & \multicolumn{3}{|c|}{$3^{e}$ adaptée } & \multirow{2}{*}{$\begin{array}{c}\text { Comparaison } \\
\text { entre } \\
3^{e} \text { générale } \\
\text { et } 3^{e} \text { adaptée } \\
\text { (test t) }\end{array}$} \\
\hline & Garçons & Filles & $\begin{array}{l}\text { Différences } \\
\text { entre garçons } \\
\text { et filles }(t)\end{array}$ & Garçons & Filles & $\begin{array}{l}\text { Différences } \\
\text { entre garçons } \\
\text { et filles }(t)\end{array}$ & \\
\hline $\begin{array}{l}\text { Sentiment de } \\
\text { proximité/distance }\end{array}$ & $\begin{array}{c}11,6 \\
(\sigma=1,7)\end{array}$ & $\begin{array}{c}11,6 \\
(\sigma=1,6)\end{array}$ & ns & $\begin{array}{c}11,4 \\
(\sigma=1,3)\end{array}$ & $\begin{array}{c}11,5 \\
(\sigma=1,2)\end{array}$ & ns & ns \\
\hline
\end{tabular}

les réponses des filles et celles des garçons. La valeur scolaire (instrumentale) de l'école est la dimension recueillant les moyennes les plus fortes. Les buts scolaires que se fixe l'élève sont: "enrichir mes connaissances ", "me perfectionner ", « apprendre de nouvelles choses ", " avoir des résultats qui me permettront de choisir un métier que j'aime ", "Avoir de bonnes notes parce que c'est important pour moi » et «accéder à des études supérieures ". En second viennent les valeurs de socialisation. Les buts poursuivis relèvent d'une recherche de contact avec le groupe de pairs. Ce sont des buts de sociabilité : « être avec des amis ", "être avec des jeunes de mon âge ", " faire de nouvelles connaissances", "être en relation avec le sexe opposé ", «m'amuser ». Et enfin viennent des valeurs hétéronomes de conformité aux attentes des autres (pairs, parents, enseignants), renvoyant à la sensibilité à l'égard du jugement d'autrui : "avoir de bonnes notes pour que mes parents et professeurs soient fiers de moi », " faire plaisir à mes parents " (voir en annexe 2). Le souci d'affirmer ou de restaurer une image de soi sociale conforme à un certain idéal de réussite scolaire est mis en avant ici.

Les résultats des tests de moyenne ne montrent aucune différence de sexe, filles et garçons accordant la même valeur à l'école. On relèvera également l'absence de différences significatives entre la filière SEGPA et la filière générale.

\section{Sentiment de proximité/distance}

D'une manière générale, le sentiment de proximité/ distance vis-à-vis de la scolarité des collégien-ne-s interrogé-e-s est en demi-teinte. En effet $71 \%$ d'entre eux affirment faire, au niveau du travail scolaire, juste ce qu'on leur demande, sans aller au-delà ; $42 \%$ se demandent parfois ce qu'ils font au collège. Malgré tout, $63 \%$ indiquent éprouver du plaisir à aller en cours. Un sentiment de solitude est ressenti par $15 \%$ d'entre eux et $6 \%$ ont l'impression que leurs parents se désintéressent de leur scolarité. Une importante majorité affirme comprendre quelles sont les attentes des enseignants, même si plus d'un tiers indique se sentir incompris par eux. Plus de la moitié des élèves interrogés avouent avoir hâte de terminer leurs études ou indiquent se sentir découragés ou bloqués face à certains apprentissages. Néanmoins, près de $80 \%$ rapportent éprouver du plaisir à réaliser certains travaux scolaires. Aucune différence significative n'a été mise en évidence, que ce soit entre les filles et les garçons ou entre les filières pour les moyennes et les écarts types (cf. le tableau 4).

\section{Différences intersexes dans le lien entre rapport à l'école et réussite scolaire}

II s'agit ici d'étudier comment, au-delà des déterminismes sociaux, chaque jeune se mobilise face aux exigences scolaires. L'hypothèse à tester concerne les effets du sens accordé à la scolarité (valeur que le sujet accorde à l'école et sentiment de proximité/ distance vis-à-vis de l'école) sur la réussite scolaire. La réussite scolaire des adolescents a été examinée sous l'angle de la performance scolaire, déterminée à partir de critères institutionnels classiques, à savoir la moyenne des notes (moyenne générale des notes aux trois trimestres). Afin d'examiner en profondeur les liens entre rapport à l'école et réussite scolaire, une série d'analyses de régression ont été réalisées séparément pour les filles et les garçons en distinguant les filières. Les résultats concernant le test du modèle sont présentés dans le tableau 5 suivant.

Nous constatons que les tests des modèles explicatifs de la réussite scolaire sont significatifs pour les élèves de filière générale (garçons et filles) et pour les garçons de SEGPA. En revanche, le modèle n'est pas validé pour les filles de SEGPA. Le tableau 6 permet d'observer les effets respectifs des différentes dimensions du rapport à l'école sur la réussite scolaire. La valeur absolue des $\beta$ traduit l'importance relative des variables explicatives.

La réussite des garçons en filière générale se caractérise par des attentes fortes en matière de valeurs 
Tableau 5. - Tests d'ajustement des modèles explicatifs de la réussite scolaire

\begin{tabular}{|c|c|c|c|c|}
\hline \multirow[b]{2}{*}{$\begin{array}{l}\text { Caractéristiques du modèle testé } \\
\text { pour chaque filière }\end{array}$} & \multicolumn{2}{|c|}{ Garçons } & \multicolumn{2}{|c|}{ Filles } \\
\hline & $\mathrm{R}^{2}$ ajusté & $\mathrm{F}$ & $\mathrm{R}^{2}$ ajusté & $\mathrm{F}$ \\
\hline Résultats pour la $3^{e}$ générale & 0,13 & $9,846^{\star * *}$ & 0,11 & $5,986^{\star \star \star}$ \\
\hline Résultats pour la $3^{\mathrm{e}}$ adaptée & 0,11 & $2,556^{\star}$ & $-0,10$ & ns \\
\hline
\end{tabular}

Note : ${ }^{*}$ significatif à $p<0,05 ;{ }^{* * *}$ significatif à $p<0,001$.

Tableau 6. - Dimensions du rapport à l'école prédictives de la réussite scolaire

\begin{tabular}{|c|c|c|c|c|c|}
\hline & \multirow[b]{2}{*}{ Variables dans le modèle } & \multicolumn{2}{|c|}{ Garçons } & \multicolumn{2}{|c|}{ Filles } \\
\hline & & $\beta$ & $\mathrm{p}$ & $\beta$ & $\mathrm{p}$ \\
\hline \multirow{4}{*}{$3^{e}$ générale } & Valeur scolaire & $0,247^{\star * *}$ & 0,000 & $0,139 *$ & 0,028 \\
\hline & Valeur sociale & 0,016 & 0,801 & $0,240^{\star \star *}$ & 0,000 \\
\hline & Valeur de conformité & $-0,151^{*}$ & 0,018 & $-0,127$ & 0,095 \\
\hline & Sentiment de proximité/distance & $0,203^{\star *}$ & 0,002 & $0,142^{*}$ & 0,020 \\
\hline \multirow{4}{*}{$3^{e}$ adaptée } & Valeur scolaire & $-0,054$ & 0,739 & & \\
\hline & Valeur sociale & 0,072 & 0,622 & & \\
\hline & Valeur de conformité & $-0,329^{*}$ & 0,019 & & \\
\hline & Sentiment de proximité/distance & $0,351^{*}$ & 0,043 & & \\
\hline
\end{tabular}

Note : * significatif à $\mathrm{p}<0,05 ;{ }^{* *}$ significatif à $\mathrm{p}<0,01 ;{ }^{* * *}$ significatif à $\mathrm{p}<0,001$.

scolaires instrumentales $(\beta=0,247, p<0,001)$, un sentiment élevé de proximité scolaire $(\beta=0,203, p<0,01)$ et de faibles attentes en matière de valeur de conformité (4) $(\beta=-0,151, p<0,05)$. Pour les garçons de SEGPA, l'analyse rend compte des relations entre la réussite scolaire et, d'une part, une faible recherche de conformité $(\beta=-0,329, p<0,05)$ et, d'autre part, une forte proximité vis-à-vis de la scolarité $(\beta=0,351$, $\mathrm{p}<0,05)$. En revanche la valeur instrumentale accordée à l'école n'a pas de pouvoir de prédiction sur la performance scolaire. Quant aux filles, à la différence des garçons, leur réussite se caractérise, en filière générale, par des attentes fortes à l'égard de l'école en matière de valeurs sociales $(\beta=0,240, p<0,001)$. Les attentes en matière de valeur instrumentale $(\beta=0,139, p<0,05)$ et un sentiment élevé de proximité scolaire $(\beta=0,142, p<0,05)$ sont aussi liés à la performance scolaire, mais dans une moindre mesure. Rappelons en revanche l'absence d'effets d'interaction significatifs pour le sous-échantillon de filles en SEGPA, marquant de fait une différence intrasexe.

\section{UNE PLUS FORTE SOCIABILITÉ CHEZ LES FILLES POUR UN MEILLEUR INVESTISSEMENT SCOLAIRE}

Nous reprenons ici les résultats les plus saillants de notre étude, à la lumière des travaux évoqués dans la première partie de cet article. Puis nous poursuivons l'analyse par une discussion sur les liens entre, d'une part, les différences observées entre garçons et filles quant aux modes de sociabilité et, d'autre part, la réussite scolaire.

\section{Que retenir de ces résultats en termes de différences et de similitudes dans les parcours scolaires?}

Notre étude permet de prendre la mesure des effets, parfois relatifs, du genre sur l'expérience scolaire. Filles et garçons ne suivent pas le même itinéraire scolaire. D'une part, les filles redoublent significativement moins que les garçons, conformément à ce qui 
est présenté dans la littérature (Bouchard \& SaintAmant, 1999 ; MEN, 2008). D'autre part, leur performance scolaire se concrétise par de meilleures notes, ce qui corrobore un constat déjà fréquemment rapporté (Baudelot \& Establet, 2007). Enfin les filles sont plus nombreuses que les garçons à aimer aller au collège. Dans ce sens, Terrail (1997) a montré que les filles investissent mieux l'école et que les garçons réinvestissent mieux leurs atouts scolaires dans leur carrière professionnelle. Néanmoins nos résultats ne permettent pas de mettre en exergue des différences de sexe en ce qui concerne le vécu au collège, la valeur accordée à l'école et le sens donné à son expérience scolaire. En effet, sur les critères objectifs communément utilisés pour caractériser la démobilisation scolaire (sécher les cours, absences et retards répétés), aucune différence significative entre les déclarations des filles et celles des garçons n'est observée. Par ailleurs, filles et garçons accordent la même valeur à l'école et ne se distinguent pas en ce qui concerne le questionnement du sens de la scolarité.

Un deuxième objectif de l'étude visait à vérifier l'existence de variations dans l'expérience scolaire des filles et des garçons scolarisés en section d'enseignement général et professionnel adapté (SEGPA). II s'avère que les différences intersexes sont encore plus estompées (moyennes, vécu et parcours ne se distinguent pas) pour les élèves de SEGPA. Ce résultat peut être rapproché des constats de Bouchard et de ses collègues (2000) concernant l'existence de traits communs aux filles et garçons présentant des difficultés scolaires graves et durables. En se démarquant, en contexte de ségrégation scolaire, des stéréotypes féminins, les filles en tirent-elles pour autant un bénéfice conséquent en termes de succès scolaire?

Enfin nos résultats montrent que les différences de trajectoire scolaire entre les filières (filière générale vs SEGPA) restent relativement faibles, pour les filles comme pour les garçons, mis à part pour la performance (moyenne des notes). Mais les inégalités sociales sont mises en lumière, les enfants des mères les moins scolarisées étant plus nombreux en filière adaptée. Ceci rejoint la série de travaux relatifs aux enjeux de l'éducation familiale sur le développement de l'enfant, qui ont souligné l'incidence culturelle et développementale déterminante et spécifique du niveau de scolarisation de la mère sur «l'adaptation sociale et scolaire" de l'enfant (Pourtois, 1979; Stevenson \& Baker, 1987 ; Prêteur \& Vial, 1997), en raison de son engagement éducatif (qualitativement et quantitativement) plus marqué que celui du père.
Contrairement aux études (notamment de I'INSEE) qui privilégient le critère socio-économique pour catégoriser le milieu social des familles, un niveau d'études élevé des parents est associé à des attitudes et conduites éducatives plus pro-actives, favorisant le développement de l'autonomie de l'éduqué dans ses différents domaines de vie (Prêteur, Lescarret \& Léonardis, 1998). Derrière ce débat du choix des indicateurs pour spécifier le milieu d'origine des élèves, c'est le statut de la variable proximale «éducation familiale » qui se joue. Le niveau d'études de la mère est ainsi bien relié à l'adaptation scolaire et à la réussite de l'enfant.

\section{Que retenir concernant les liens entre rapport à l'école et performance scolaire?}

Alors que les filles et les garçons présentent des similitudes du point de vue des valeurs qu'ils accordent à l'école et du sens accordé à l'expérience scolaire, notre étude montre qu'ils développent des stratégies d'investissement différentes. Pour les garçons en filière générale, trouver ou donner du sens à sa présence à l'école tout en développant des valeurs scolaires instrumentales est un enjeu important de la réussite scolaire. Chez leurs camarades de sexe féminin, le rapport, à la fois plus fréquemment épistémique à l'apprendre et plus internalisé, s'accommode davantage aux pratiques sociales et aux valeurs sociales de l'école (Prêteur, Constans \& Féchant, 2004). Les relations à l'autre, qui s'inscrivent dans le cadre familial et scolaire, mais surtout amical, qu'elles soient harmonieuses ou conflictuelles, sources de plaisir ou de désintérêt, sont très fréquemment évoquées et valorisées par les adolescentes. Elles apparaissent en général plus « compétentes socialement " que leurs homologues masculins (Mallet, 2003). De ce point de vue, le collège offre une réelle opportunité d'entretenir ces relations sociales et de satisfaire leurs attentes plus importantes de sociabilité. Ces observations sont attestées aussi bien auprès de collégiennes ordinaires (Prêteur, Constans \& Féchant, 2004) que d'adolescentes à « haut potentiel » (Villatte, Léonardis \& Prêteur, 2009), ou encore en réussite paradoxale au regard de leur situation sociofamiliale (depuis un demi-siècle, de nombreuses études ont établi le lien entre le niveau socioculturel des familles et le niveau de réussite scolaire de leurs enfants, cf. notamment Safont-Mottay \& Lescarret, 2008). Au-delà de ces constats et des différences sociologiques, ces situations paradoxales (de réussite ou d'échec) nous interrogent sur l'origine des processus qui concourent à l'investissement scolaire. 
Les garçons en filière adaptée qui réussissent le mieux sont ceux qui arrivent à trouver un sens à leur présence à l'école (fort sentiment de proximité scolaire), tout en développant un rapport à l'apprendre internalisé (faible recherche de conformité). Sur ces premiers points, ces élèves de SEGPA sont proches des garçons en filière générale, les différences entre les sexes, et certainement entre les filières, se faisant sur la recherche de buts scolaires (caractéristique des garçons de la filière générale) et de buts de contacts avec le groupe de pairs (caractérisant les filles de la filière générale). L'absence d'interaction significative entre les dimensions du rapport à l'école et la performance chez les filles ne manque pas ne nous interpeller. Rappelons toutefois que l'effectif de notre population d'élèves en SEGPA est réduit (69 garçons et 49 filles), bien qu'il reflète la différence de proportion entre filles et garçons dans cette filière (MEN, 2009). La mise en œuvre de stratégies d'investissement semble plus difficile et laisse à penser que la scolarisation en filière adaptée est plus stigmatisante pour les filles de notre étude que pour les garçons. On peut, à ce sujet, regretter avec Bouchard et ses collègues (2000) qu'un trop petit nombre de travaux se préoccupe des besoins et des problèmes identitaires des filles en difficulté scolaire, alors qu'un plus grand nombre vise les garçons en rupture avec l'école.

\section{Quelle est la part de la socialisation différenciée à l'école ?}

L'étude met en exergue une caractéristique communément observée dans la littérature : les filles, dans la filière générale, valorisent plus et sont plus investies dans les relations sociales et communicatives (Claes, 2003). Elles mettent en avant des valeurs de socialisation et des buts de sociabilité («être avec des amis ", "faire de nouvelles connaissances ", "être en relation avec le sexe opposé », etc.) Ces qualités sont liées « avec plusieurs aspects de la personnalité, tels que l'estime de soi et les capacités d'adaptation psychologique » (Claes, 2003, p. 102). Elles se retrouvent dans le care, non pas conçu comme une disposition psychologique qui serait inhérente au développement psychocognitif des femmes, mais reposant sur une série d'expériences ou d'activités (Molinier, Laugier \& Paperman, 2009).

La socialisation différenciée contribue sans doute à expliquer l'accent mis de façon bien plus marquée sur la sphère relationnelle dans notre échantillon féminin. On ne saurait toutefois réduire cet intérêt pour les relations sociales à une simple conformation aux stéréo- types de sexe. Convoquer uniquement le processus de reproduction des rapports sociaux de sexe selon lequel les filles se soumettraient passivement aux stéréotypes qui leur sont assignés socialement, ce qui leur conférerait un avantage sur le plan scolaire, tend à déprécier la réussite des filles (Bouchard \& Saint-Amant, 1999). Cela sous-entend en effet que celles-ci réussiraient mieux parce qu'elles sont dociles et parce que l'école favorise la passivité. De plus il n'est fait aucune place, dans ce type d'analyse, au rôle joué par l'élève dans la prise en charge de son propre cheminement scolaire et dans la construction de son identité. Or les filles développent très tôt des stratégies de communication (Gagnon, 1999 ; Ferrez, 2006), reçoivent davantage de feedback verbaux des professionnels du jeune enfant que les garçons (Honing \& Wittmer, 1982). Les recherches stipulent que les filles seraient plus précocement encouragées à être conscientes des besoins d'autrui (Molinier, Laugier \& Paperman, 2009), ce qui les inciterait à développer des attitudes qui privilégient la coopération et la prise en compte du point de vue d'autrui. Comme le développent ces auteurs, les filles et les femmes articulent fréquemment le domaine de la subjectivité et des affects avec celui du travail (scolaire ou professionnel). Par cette posture identitaire, il est par conséquent concevable qu'elles investissent plus fortement les relations sociales, la communication (verbale tout spécialement) et que ces expériences et activités, largement investies, se concrétisent également par des compétences pour apporter des réponses concrètes aux besoins et attentes des autres (travail domestique, de soin, d'éducation, de soutien ou d'assistance, d'échange même). Chez les garçons, les comportements d'affirmation, d'autonomie et de compétition seraient davantage renforcés (Villatte, Léonardis \& Prêteur, 2009). Mais l'adhésion impérative aux rôles de sexe semble augmenter chez eux le risque d'un sous-développement des capacités sociales. Comme l'a montré Mallet (2003), les garçons qui, dans les interactions sociales avec leurs pairs, se conforment aux rôles typiques de leur sexe rencontrent des difficultés à établir une relation d'amitié fondée sur « l'intimité et l'engagement émotionnels » et se privent d'un soutien émotionnel des plus efficaces. Au-delà même du domaine scolaire, les nouveaux modes de communication chez les adolescents (Pasquier, 2005) accusent cette dissociation de la sphère de l'intime et du collectif plus marquée chez les garçons. Les filles, là encore, par leur usage différencié des modes de communication à distance, transfèrent plus aisément dans le domaine scolaire les capacités sociales développées dans le domaine extrascolaire (cf. la problématique du care évoquée plus haut). 


\section{CONCLUSION ET PERSPECTIVES}

Cette étude met en évidence des liens entre le sens accordé à la scolarité et l'expérience scolaire chez les adolescents à la fin du collège. La valeur accordée à l'école s'avère particulièrement importante dans la compréhension de la manière dont l'élève, porteur d'une histoire, se pense comme apprenant et conçoit les savoirs et leurs finalités. Ainsi les élèves qui adhèrent à des valeurs autonomes, ou savent qu'il faut s'y référer, se mobilisent davantage dans le travail scolaire. Mais le collège est aussi un lieu de convivialité, de sociabilité et les relations interpersonnelles, essentielles, nourrissent la socialisation horizontale et participent à l'établissement d'une culture scolaire favorisant aussi l'appropriation du savoir scolaire. Ce résultat ouvre une piste de réflexion sur les fonctions du groupe de pairs - réconfort, soutien social, affectivité - dans la mobilisation scolaire, et cela tout particulièrement dans les filières faiblement féminisées. Les différences observées selon le sexe incitent à étudier plus précisément le rôle joué par les relations d'affinité entre adolescents dans la représentation de l'école et des buts scolaires. Si l'adolescent attend soutien et reconnaissance des adultes (parents et enseignants) dans sa vie scolaire, il est également souvent orienté et influencé par ses pairs, selon ses réseaux d'affiliation, dans son rapport à l'école et au savoir scolaire. Socialisations verticales et horizontales contribuent donc à construire, au quotidien, ses attentes, valeurs, conduites d'élève et stratégies identitaires. Considérés encore récemment comme rares et annexes, les phénomènes d'éloignement des jeunes face à l'école et aux apprentissages scolaires deviennent aujourd'hui des sujets de préoccupation majeure.

Ainsi donc, par le jeu très subtil des interactions entre enseignants et élèves (Zaidman, 1996 ; DuruBellat, 2005 ; Dafflon Novelle, 2006 ; Ferrez, 2006) et des interactions entre pairs, filles et garçons vivent à l'école des expériences sociales n'offrant pas le même type d'opportunité de réalisation de soi. Mais rappelons que l'école est à l'image d'un modèle plus général de société. Et si l'école entretient et parfois même consolide les stéréotypes de sexe, elle n'a pas de pouvoir exclusif. C'est pourquoi il nous semble important de poursuivre l'analyse du registre identitaire par l'étude des tensions afférentes au fait de "grandir" et de construire une identité sexuée au moment de l'entrée dans l'adolescence. Nous assistons actuellement à une multiplication des modèles de socialisation (images culturelles véhi- culées par les médias, par les réseaux sociaux, par Internet...) touchant précocement les enfants, et en particulier les filles (Pasquier, 2005 ; Monnot, 2009). Des contradictions fortes peuvent alors apparaître entre exigences parentales, exigences scolaires et conformité avec des modèles très prégnants de la masculinité et de la féminité imposés par les médias dans nos sociétés contemporaines. Le rapport à l'école et la primauté des savoirs scolaires pour les futur-e-s collégien-ne-s et lycéen-ne-s en seront-ils affectés?

À partir de cette analyse, quelques recommandations en termes de politiques éducatives peuvent être envisagées. Les comportements des jeunes sont marqués par les rôles sociaux empruntés aux modèles que leur propose la société adulte ; l'école ellemême n'échappe pas aux stéréotypes sociaux de sexe. Ces constats remettent-ils pour autant en cause le principe de la co-éducation des filles et des garçons dans un même lieu scolaire ? L'école, mise au défi de contribuer à la construction de l'identité des adolescent-e-s dans un environnement marqué par les stéréotypes, se doit de proposer aux jeunes un accompagnement qui sache les rendre acteurs dans le processus de découverte de l'altérité. La mixité ne peut pas seulement se décréter. Pour la construire au quotidien, il importe que les différents partenaires éducatifs, et tout particulièrement les enseignants (dans la mesure où il leur incombe d'organiser et de structurer les activités scolaires), prennent préalablement conscience des stéréotypes de sexe que nous véhiculons inévitablement. Une piste consiste ensuite à diversifier des activités qui fassent appel aux compétences complémentaires des unes et des autres, à savoir les capacités de communication privilégiées par les filles et les savoir-faire instrumentaux plus valorisés par les garçons. La reconnaissance simultanée de ces deux registres s'avère nécessaire afin de favoriser à l'adolescence la coopération, la réciprocité des échanges entre pairs et l'ouverture à l'autre.

Claire Safont-Mottay mottay@univ-tlse2.fr

Psychologie du développement et processus de socialisation, université Toulouse-Le Mirail-Toulouse 2

Nathalie Oubrayrie-Rousse

Psychologie du développement et processus de socialisation, université Toulouse-Le Mirail-Toulouse 2

Yves Prêteur

Psychologie du développement et processus de socialisation, université Toulouse-Le Mirail-Toulouse 2 


\section{NOTES}

(1) Au-delà de la scolarité à l'école élémentaire et sur avis d'une commission, les élèves non handicapés relevant de l'enseignement spécialisé peuvent être admis dans la section spécialisée de certains collèges (appelée depuis 1989 section d'enseignement général et professionnel adapté).

(2) Selon la nomenclature INSEE.
(3) Les liaisons sont appréciées non plus seulement prises deux à deux, mais en tenant compte des interactions.

(4) Le signe négatif devant le coefficient $\beta$ indique une relation significative négative entre la valeur de conformité et la réussite scolaire.

\section{BIBLIOGRAPHIE}

BAUDELOT C. \& ESTABLET R. (2007). Quoi de neuf chez les filles ? Entre stéréotypes et libertés. Paris : Nathan.

BERNDT T., LAYCHAK A. \& PARK K. (1990). « Friend's influence on adolescents' academic achievement motivation: An experimental study ". Journal of Educational Psychology, vol. 82, $\mathrm{n}^{\circ}$ 4, p. 664-670.

BOUCHARD P. \& SAINT-AMANT J.-C. (1999). Garçons et filles. Stéréotypes et réussite scolaire. Montréal : Éd. du remue-ménage.

BOUCHARD P., SAINT-AMANT J.-C. \& TONDREAU J. (1998). "Effets de sexe et de classe sociale dans l'expérience scolaire de jeunes de quinze ans ". Cahiers québécois de démographie, vol. 27, $\mathrm{n}^{\circ} 1$, p. 95-120.

BOUCHARD P. \& SAINT-AMANT J.-C., GAUVIN M. et al. (2000). «Familles, écoles et milieu populaire ». Études et recherches du CRIRES, vol. 5, $\mathrm{n}^{\circ} 1$.

CHARLOT B., BAUTIER É. \& ROCHEX J.-Y. (1992). École et savoir dans les banlieues... et ailleurs. Paris : Armand Colin.

CHEVET M. (2006). "L'impact du genre dans la relation entre enseignant-e-s et apprenant-e-s ». Études de linguistique appliquée, vol. 142, n² 2, p. 163-174.

CLAES M. (2003). L'univers social des adolescents. Montréal : Presses de l'Université de Montréal.

COSLIN G. (2007). La socialisation de l'adolescent. Paris Armand Colin.

COURTINAT-CAMPS A. \& PRÊTEUR Y. (2010). « Expérience scolaire à l'adolescence: quelles différences entre les filles et les garçons ? " In V. Rouyer, S. Croity-Belz \& Y. Prêteur (dir.), Genre et socialisation de l'enfance à l'âge adulte. Expliquer les différences, penser l'égalité [à paraître]. Toulouse : Érès, p. 91-105.

DAFFLON NOVELLE A. (2006). Filles-garçons. Socialisation différenciée? Grenoble: Presses universitaires de Grenoble.

DUBET F. \& MARTUCCELLI D. (1996). À l'école. Sociologie de l'expérience scolaire. Paris : Éd. du Seuil.

DUMORA B. (2001). "Les intentions d'orientation et leur argumentation". L'orientation scolaire et professionnelle, vol. $30, \mathrm{n}^{\circ} 2$, p. 148-165.

DURU-BELLAT M. (2005). L'école des filles. Quelle formation pour quels rôles sociaux? Paris: L'Harmattan.

DURU-BELLAT M. \& MARIN B. (2009). La mixité à l'école : filles et garçons. Champs-sur-Marne: CRDP de l'académie de Créteil.

DURU-BELLAT M. \& ZANTEN A. van (2009). Sociologie du système éducatif. Les inégalités scolaires. Paris: PUF.
FELOUZIS G. (1993). "Interactions en classe et réussite scolaire: une analyse des différences filles-garçons ". Revue française de sociologie, $n^{\circ} 34$, p. 199-122.

FERREZ E. (2006). « Éducation préscolaire : filles et garçons dans les institutions de la petite enfance ». In A. Dafflon Novelle (dir.), Filles-garçons. Socialisation différenciée ? Grenoble: Presses universitaires de Grenoble, p. 69-83.

FOREST L. (1992). "L'école primaire "mixte" : une école pour les filles, une école pour les garçons ". In C. Baudoux \& C. Zaidman (dir.), Égalité entre les sexes. Mixité et démocratie. Paris : L'Harmattan.

FULIGNI A., ECCLES J., BARBER B. \& CLEMENTS P. (2001). "Early adolescent peer orientation and adjustment during high school ". Developmental Psychology, vol. 37, $\mathrm{n}^{\circ} 1$, p. 28-36.

GAGNON C. (1999). Pour réussir dès le primaire. Filles et garçons face à l'école. Montréal: Éd. du remueménage.

HONING A. \& WITTMER D. (1982). «Teacher questions to male and female toddlers". Early Child Development and Care, vol. 9, $\mathrm{n}^{\circ} 1$, p. 19-32.

JARLÉGAN A. \& TAZOUTI Y. (2007). « Jugements des enseignants et représentations liées aux différences de sexe et d'appartenance sociale des élèves ". Symposium Genre et éducation, actes du Congrès International AREF, Strasbourg. Disponible sur Internet à l'adresse : <http://www.congresintaref.org/actes pdf/AREF2007 Annette_JARLEGAN_095.pdf> (consulté le 10 novembre 2009).

JELLAB A. (2001). Scolarité et rapport aux savoirs en lycée professionnel. Paris : PUF.

LADD G. (1990). « Having friends, keeping friends, making friends, and being liked by friends in the classroom: Predictor of children's early school adjustment ». Child Development, vol. 61, n 4, p. 1081-1100.

LÉONARDIS M. de \& PRÊTEUR Y. (2007). « Expérience scolaire, estime de soi et valeur accordée à l'école à l'adolescence ». In A. Florin \& P. Vrignaud (dir.), Les effets des dimensions conatives en éducation. Personnalité, motivation, estime de soi, compétences sociales. Rennes: PUR, p. 31-45.

LÉONARDIS M. de, CAPDEVIELLE V. \& PRÊTEUR Y. (2006). "Sens de l'orientation vers l'apprentissage chez les apprentis de niveau V: entre expérience scolaire et rapport à l'avenir ". L'orientation scolaire et professionnelle, vol. $35, n^{\circ} 1$, p. 5-27.

LEVESQUE S. (2001). Valeur accordée à l'école, estime de soi et performance scolaire à l'adolescence. Thèse 
de doctorat, psychologie, université Toulouse-Le MirailToulouse 2.

MALLET P. (2003). "Amitié, intimité émotionnelle et rôles de sexe à l'adolescence ". Pratiques psychologiques, $\mathrm{n}^{\circ} 3$, p. 39-48

MALRIEU P. (1980). "Genèse des conduites d'identité ". In P. Tap (dir.), Identité individuelle et personnalisation. Toulouse : Privat, p. 39-51.

MARRY C. (2003). Les paradoxes de la mixité filles-garçons à l'école. Perspectives internationales. Rapport pour le PIREF et conférence du 16 octobre 2003 au ministère de l'Éducation nationale. Disponible sur Internet à l'adresse suivante : <http://back.ac-rennes.fr/orient/ egalchanc/rapmixite22103.pdf> (consulté le 30 avril 2010).

MARRY C. (2004). "Mixité scolaire : abondance des débats, pénurie des recherches". Travail, genre et sociétés, $\mathrm{n}^{\circ} 11$, p. 189-194.

MICHINOV E. (2004). " L'influence des relations entre élèves ". Sciences humaines, $\mathrm{n}^{\circ} 45$, hors-série, p. 52-55.

MILLET M. \& THIN D. (2005). Ruptures scolaires. L'école à l'épreuve de la question sociale. Paris : PUF.

MINISTÈRE DE L'ÉDUCATION NATIONALE (2008). Filles et garçons à l'école : sur le chemin de l'égalité. Paris : ministère de l'Éducation nationale.

MINISTĖRE DE L'ÉDUCATION NATIONALE (2009). Repères et références statistiques sur les enseignements, la formation et la recherche. Paris: Direction de l'évaluation et de la prospective.

MOLINIER P., LAUGIER S. \& PAPERMAN P. (2009). Qu'estce que le care ? Souci des autres, sensibilité, responsabilité. Paris : Payot.

MONNOT C. (2009). Petites filles d'aujourd'hui. L'apprentissage de la féminité. Paris : Éd. Autrement.

MOSCONI N. (1999). "Les recherches sur la socialisation différentielle des sexes à l'école ». In Y. Lemel \& B. Roudet (dir.), Filles et garçons jusqu'à l'adolescence. Socialisation différentielle. Paris : L'Harmattan.

MOSCONI N. (2001). « Comment les pratiques enseignantes fabriquent de l'inégalité entre les sexes". Les dossiers des sciences de l'éducation, $\mathrm{n}^{\circ}$ 5, p. 97-109.

OUBRAYRIE-ROUSSEL N. \& SAFONT-MOTTAY C. (2007). "Adaptation scolaire et troubles des conduites à l'adolescence $" 4^{\mathrm{e}}$ Congrès de psychologie de la santé de langue française, Toulouse.

PASQUIER D. (2005). Cultures lycéennes. La tyrannie de la majorité. Paris : Éd. Autrement.

POTVIN P., PARADIS L. \& POULIOT B. (2000). "Attitudes des enseignants de maternelle selon le sexe des élèves ". Revue des sciences de l'éducation, vol. 26 , $\mathrm{n}^{\circ} 1$, p. $35-54$.

POURTOIS J.-P. (1979). Comment les mères enseignent à leur enfant. Paris: PUF.

PRÊTEUR Y. \& VIAL B. (1997). « Rapports à l'écrit et à l'école de la famille et de l'enfant en $1^{\text {re }}$ année du cycle des apprentissages fondamentaux ». In C. Barré de Miniac \& B. Lété (dir.), L'illettrisme. De la prévention chez l'enfant aux stratégies de formation chez l'adulte. Bruxelles : De Boeck, p. 103-127.
PRÊTEUR Y., CONSTANS S. \& FÉCHANT H. (2004). «Rapport au savoir et (dé)mobilisation scolaire chez des collégiens de troisième ". Pratiques psychologiques, $n^{\circ} 10$, p. 119-132.

PRÊTEUR Y., LESCARRET O. \& LÉONARDIS M. de (1998). "Family education, parents-child interactions and child development ». European Journal of Psychology of Education, vol. 13, n 4 , p. 461-474.

RAÏD L (2009). "Care et politique chez Jean Tronto". In P. Molinier, S. Laugier \& P. Paperman, Qu'est-ce que le care? Souci des autres, sensibilité, responsabilité. Paris : Payot.

ROCHEX J.-Y. (1995). Le sens de l'expérience scolaire. Paris : PUF.

ROUYER V. (2007). La construction de l'identité sexuée. Paris : Armand Colin.

ROUYER V. \& ZAOUCHE-GAUDRON C. (2006). " La socialisation des filles et des garçons au sein de la famille: enjeux pour le développement ». In A. Dafflon Novelle (dir.), Filles-garçons. Socialisation différenciée ? Grenoble : Presses universitaires de Grenoble, p. 27-54.

SAFONT-MOTTAY C. \& LESCARRET O. (2008). «Éducation familiale et mobilisation scolaire des adolescents en milieu défavorisé ». In G. Pithon, C. Philip-Asdih \& S. Larivée (dir.), Construire une "communauté éducative ». Un partenariat famille-école-association. Bruxelles : De Boeck, p. 91-110.

STEVENSON D. \& BAKER D. (1987). "The family-school relation and the child's school performance". Child Development, vol. 58, $\mathrm{n}^{\circ}$ 5, p. 1348-1357.

TAP P. (1985). Masculin et féminin chez l'enfant. Toulouse : Privat.

TAP P. \& ZAOUCHE-GAUDRON C. (1999). «Identités sexuées, socialisation et développement de la personne ». In Y. Lemel \& B. Roudet, Filles et garçons jusqu'à l'adolescence. Socialisations différentielles. Paris : L'Harmattan, p. 25-56.

TERRAIL J.-P. (1992a). "Réussite scolaire : la mobilisation des filles ». Sociétés contemporaines, $\mathrm{n}^{\circ}$ 11-12, p. 53-89.

TERRAIL J.-P. (1992b). "Parents, filles et garçons face à l'enjeu scolaire". Éducation et formations, $n^{\circ} 30$, p. 3-11.

TERRAIL J.-P. (1997). «La supériorité scolaire des filles». In J.-P. Terrail (dir.), La scolarisation de la France. Critique de l'état des lieux. Paris : La Dispute.

VILLATTE V., LÉONARDIS M. de \& PRÊTEUR Y. (2009). « Le concept de soi des lycéen(ne)s à haut potentiel. Perspectives comparatives filles/garçons ". Recherches \& éducation, $n^{\circ} 2$, p. 201-226.

ZAIDMAN C. (1996). La mixité à l'école primaire. Paris: L'Harmattan.

ZANTEN A. van (2001). "Les amitiés et la sociabilité des adolescents de banlieue: autonomie, intégration et ségrégation ». In A. Braconnier, C. Chiland \& M. Choquet (dir.), Secrets et confidents au temps de l'adolescence. Paris : Masson, p. 57-76.

ZAZZO B. (1993). Féminin et masculin à l'école et ailleurs. Paris: PUF. 
Annexe 1. - Tableau des caractéristiques sociobiographiques des élèves de $3^{e}$ générale et $3^{\mathrm{e}}$ adaptée

\begin{tabular}{|c|c|c|c|c|}
\hline \multicolumn{2}{|c|}{$3^{e}$ générale } & Garçons $(n=322)$ & Filles $(n=241)$ & Total $(n=563)$ \\
\hline \multicolumn{2}{|r|}{ Âge } & $\begin{array}{c}m=14,7 \text { ans } \\
(\sigma=0,73)\end{array}$ & $\begin{array}{c}\mathrm{m}=14,7 \text { ans } \\
(\sigma=0,76)\end{array}$ & $\begin{array}{c}\mathrm{m}=14,7 \text { ans } \\
(\sigma=0,75)\end{array}$ \\
\hline \multirow{3}{*}{$\begin{array}{l}\text { Scolarité } \\
\text { de la mère }\end{array}$} & $3^{e}$, CAP ou BEP & $38,5 \%$ & $27,4 \%$ & $33,6 \%$ \\
\hline & Niveau bac & $31,5 \%$ & $37,3 \%$ & $34,1 \%$ \\
\hline & Supérieur & $30,0 \%$ & $35,3 \%$ & $32,3 \%$ \\
\hline \multirow{4}{*}{$\begin{array}{l}\text { Profession } \\
\text { du père }\end{array}$} & Très défavorisée & $23,0 \%$ & $23,0 \%$ & $23,0 \%$ \\
\hline & Défavorisée & $37,0 \%$ & $30,0 \%$ & $34,2 \%$ \\
\hline & Favorisée & $19,0 \%$ & $23,0 \%$ & $20,3 \%$ \\
\hline & Très favorisée & $21,0 \%$ & $24,0 \%$ & $22,6 \%$ \\
\hline \multicolumn{2}{|c|}{$3^{\mathrm{e}}$ adaptée } & Garçons $(n=69)$ & Filles $(n=49)$ & Total $(n=118)$ \\
\hline \multicolumn{2}{|r|}{ Âge } & $\begin{array}{c}m=14,7 \text { ans } \\
(\sigma=0,76)\end{array}$ & $\begin{array}{c}m=14,7 \text { ans } \\
(\sigma=0,74)\end{array}$ & $\begin{array}{c}\mathrm{m}=14,7 \text { ans } \\
(\sigma=0,75)\end{array}$ \\
\hline \multirow{3}{*}{$\begin{array}{l}\text { Scolarité } \\
\text { de la mère }\end{array}$} & $3^{e}$, CAP ou BEP & $71,9 \%$ & $65,5 \%$ & $68,9 \%$ \\
\hline & Niveau bac & $21,9 \%$ & $24,1 \%$ & $23,0 \%$ \\
\hline & Supérieur & $6,3 \%$ & $10,3 \%$ & $8,2 \%$ \\
\hline \multirow{4}{*}{$\begin{array}{l}\text { Profession } \\
\text { du père }\end{array}$} & Très défavorisée & $35,4 \%$ & $31,4 \%$ & $33,7 \%$ \\
\hline & Défavorisée & $50,0 \%$ & $40,0 \%$ & $45,8 \%$ \\
\hline & Favorisée & $12,5 \%$ & $25,7 \%$ & $18,1 \%$ \\
\hline & Très favorisée & $2,1 \%$ & $2,9 \%$ & $2,4 \%$ \\
\hline
\end{tabular}

Annexe 2. - Liste des 13 items (en italique) retenus parmi les 20 items initiaux du questionnaire concernant la valeur accordée à l'école (Levesque, 2001 ; Léonardis \& Prêteur, 2007)

Je veux...

1) Accéder à des études supérieures (valeur scolaire)

2) Apprendre de nouvelles choses (valeur scolaire)

3) Avoir de bonnes notes parce que c'est important pour moi (valeur scolaire)

4) Avoir de bonnes notes pour que mes parents et professeurs soient fiers de moi (valeur de conformité)

5) Avoir des résultats qui me permettront de choisir un métier que j'aime plus tard (valeur scolaire)

6) Avoir un métier après le bac

7) Enrichir mes connaissances (valeur scolaire)

8) Entrer en relation avec des personnes de sexe opposé (valeur sociale)

9) Être avec des ami(e)s (valeur sociale)
10) Être avec des gens qui partagent les mêmes idées que moi

11) Être avec des jeunes de mon âge (valeur sociale)

12) Exercer une profession respectable plus tard

13) Faire de nouvelles connaissances (valeur sociale)

14) Faire plaisir à mes parents (valeur de conformité)

15) M'amuser (valeur sociale)

16) M'éloigner du foyer familial

17) Montrer aux autres que je ne suis pas bête

18) Participer à des activités qui m'apprennent quelque chose

19) Participer à des activités scolaires intéressantes

20) Perfectionner mes connaissances dans certaines matières (valeur scolaire) 